To appear in the Journal of Modern Optics Vol. 00, No. 00, 00 Month 20XX, 1-12
This is an Accepted Manuscript of an article published by Taylor \& Francis in Journal of Modern Optics on 3 June 2015, available online: http:// www.tandfonline.com/10.1080/09500340.2015.1047422 http:// authorservices.taylorandfrancis.com/sharing-your-work/ )Publisher journal website 23/9/2016

\title{
Electron angular distributions of noble gases in sequential two-photon double ionization
}

 \\ Deinert $^{\mathrm{b}}$, L. Glaser ${ }^{\mathrm{b}}$, O. Al-Dossary ${ }^{\mathrm{f}}$, A. Ehresmann ${ }^{\mathrm{e}}$, A. S. Kheifets ${ }^{\mathrm{g}}$, and J. Viefhaus ${ }^{\mathrm{b}}$ \\ ${ }^{a}$ Fritz-Haber-Institut der Max-Planck-Gesellschaft, Faradayweg 4-6, 14195 Berlin, Germany; \\ ${ }^{b}$ Deutsches Elektronen-Synchrotron DESY, Notkestraße 85, 22607 Hamburg, Germany; \\ ${ }^{c}$ European XFEL GmbH, Albert-Einstein-Ring 17-19, 22761 Hamburg, Germany; \\ ${ }^{d}$ PULSE at Stanford, 2575 Sand Hill Road, Menlo Park, 94025 California, USA; \\ ${ }^{e}$ Universität Kassel, Institut für Physik und CINSaT, Heinrich-Plett-Str. 40, 34132 Kassel, Germany; \\ ${ }^{f}$ Physics Department, King Saud University, Riyadh 11451, Saudi Arabia; \\ ${ }^{g}$ Research School of Physics and Engineering, The Australian National University, Canberra ACT 0200, \\ Australia \\ (Received 00 Month 20XX; final version received 00 Month 20XX)
}

\begin{abstract}
We present an angle resolved study of photoelectrons emitted from ions of the noble gases neon, argon and krypton by means of time-of-flight spectroscopy. The ionic targets are generated in a sequential two-photon process induced by the free-electron laser FLASH. Values of the anisotropy parameters $\beta_{2}$ and $\beta_{4}$ are derived from electron angular distribution measurements in the photon energy range from 38 to $91 \mathrm{eV}$ and compared to recent theoretical calculations.
\end{abstract}

\section{Introduction}

The investigation of light-matter interaction allows for a widespread insight into physical and chemical properties of atoms, molecules, clusters and condensed matter. Synchrotron radiation facilities have enabled exploration of the electronic structure of targets in photoionization experiments during the last decades. Regarding sequential and simultaneous photoionization numerous synchrotron radiation studies revealed valuable information to understand higher order ionization by a single photon $[1,2]$. For example, the single-photon direct double ionization of rare gases is associated with the simultaneous ejection of two electrons driven by electron correlation. In this process, the two emitted electrons can share the excess energy of the photon in a continuous kinetic energy distribution [3, 4]. Inner shell ionization with subsequent Auger decay is a second example for multiple ionizations triggered by a single photon. The Auger decay is an intra-atomic relaxation process due to Coulomb interaction [5]. This decay can be regarded as a relaxation induced sequential double ionization process [6]. The Auger process itself exhibits simultaneous and sequential character if two or even more electrons are involved in the decay. In electron-electron coincidence experiments continuous kinetic energy structures of direct double Auger decays have been observed in the case of neon [7] and argon [8], whereas for the heavier rare gases krypton and xenon sequential cascade Auger processes are found to be dominant $[9,10]$. In recent years, originated by the advent of free-electron lasers [11-14], ultra-intense and ultra-short XUV light pulses became available. Based on these novel possibilities, sequential and simultaneous double ionization involving absorption of

${ }^{*}$ Corresponding author. Email: gregor.hartmann@desy.de 
two or more photons can be studied which can give information complementary to the synchrotron results. Light-matter interaction with multiple photons of photon-energies ranging from the VUV into the hard X-ray regime, leverages the exploration of the creation of highly charged ions. In this regard, many experimental as well as theoretical investigations have been performed concerning fundamental multi-photon ionization processes [15-20]. In most of these studies, the experimental method was restrained to the detection of ions which helped to develop models of pathways up to the highest charged states as for example $\mathrm{Xe}^{36+}[15]$. Up to now, much less is known about the electronic symmetry of ionic targets. Therefore, we used angle resolved photoelectron spectroscopy to investigate the photoelectron emission and dynamics of multi-photon processes. In particular we were interested in how the photoionization dynamics influence the angular distribution of the photoelectrons in these ionic systems. Whereas in single-photon ionization the photoemission angular distribution is characterized by a single anisotropy parameter, higher order contributions with according parameters have to be added in the description of multi-photon ionization. Anisotropy patterns of that kind are known from studies of polarized targets which were prepared and ionized by two different light sources (HHG and synchrotron radiation) [21-23]. In those experiments, the values of the anisotropy parameters $\beta_{2}$ and $\beta_{4}$ of the two-step process and differences for partial waves of different angular momentum have been determined.

In this context, Cooper- and correlative minima in the photoelectron partial cross sections are other important aspects regarding properties of the electronic structure and have been investigated for single-photon single ionization in the past [1]. Cooper-minima are known to arise from the decrease of the overlap integral of the bound and the continuum electronic state of the outgoing electron [24] whereas correlative minima are caused by the transition between correlation dominated photoelectron emission at lower energies and quasi-single electron emission at higher energies [25, 26]. Reflections of these intensity variations along photon energy are also exhibited in the angular distribution of the photoelectron emission leading to a variation of the anisotropy parameter $\beta$. Such radial integral effects should actually not show major differences for different angular momentum coupling multiplets of the same electron configuration. This is in fact the case for the single-photon ionization of all rare gases. In a sequential two-photon ionization process of noble gases the second step can be regarded as the ionization of an open shell system with an anisotropic Coulomb field. Hence, anisotropic final state interactions are expected to occur [27]. Some recent calculations predict indeed large effects with respect to the higher order anisotropy parameter $\beta_{4}$, showing completely different behaviour in the region of the Cooper-minima for the different multiplets [28-30]. The intense free-electron laser radiation is capable of creating a dense ionic target which can be further ionized within the same light pulse. With this experimental studies are feasible which can reveal if these interactions constitute a large effect in double ionization. In the past, such studies were challenging to realize since they demand ion-sources which are typically limited to a relatively low target density and accordingly, prohibitively long beamtimes. Using the highly intense VUV radiation of the Free-electron LASer in Hamburg (FLASH) at DESY in Hamburg, Germany $[11,31]$, we performed measurements of electron angular distributions in sequential two-photon double ionization of the noble gases neon, argon and krypton. In this report, we focus on the higher order angular distributions in order to demonstrate the effect of multiple dipole interactions on the angular distribution of photoelectrons.

\section{Theory}

A detailed description of the theoretical model of the angular distribution of photoelectron emission on sequential two-photon double ionization is given in the related article of this issue [A.N. GrumGrzhimailo et al. in this journal]. In short, the angular distribution of a photoelectron in the dipole approximation is described by the differential cross section which is a partial wave expansion in terms of Legendre polynomials $P_{i}$ : 


$$
\frac{d \sigma}{d \Omega}=\frac{\sigma}{4 \pi} \sum_{i=0}^{\infty} a_{2 i} P_{2 i}(\cos \theta)
$$

In equation (1), $\sigma$ denotes the cross section of the photoionization process transferring the target from the neutral initial to the ionic final state and $\theta$ is the angle of the electron emission direction with respect to the linear polarization of the ionizing radiation. In the case of single photoionization, the angular distribution is given by the second order term of the expansion which includes the anisotropy parameter $\beta_{2}$ [32]:

$$
\frac{d \sigma}{d \Omega}=\frac{\sigma}{4 \pi}\left(1+\beta_{2} P_{2}(\cos \theta)\right)
$$

For multi-photon ionization, higher order terms have to be included according to the number of photons involved in the process. In the case of a two-photon ionization, the angular distribution can be described by a formula up to a Legendre polynomial of fourth order:

$$
\frac{d \sigma}{d \Omega}=\frac{\sigma}{4 \pi}\left(1+\beta_{2} P_{2}(\cos \theta)+\beta_{4} P_{4}(\cos \theta)\right)
$$

Here, the additional term including the fourth order anisotropy parameter $\beta_{4}$ term is directly proportional to the alignment of the residual ionic core created by the first ionization step [28, 33].

\section{Experimental Setup}

Figure 1 shows a sketch of the experimental setup used in our measurement campaigns at FLASH. In a spherical vacuum chamber, up to 22 independently working electron time-of-flight spectrometers have been mounted at different angles with respect to the horizontal polarization axis of the incoming light. However, due to restrictions in the data acquisition system only a maximum number of 16 eTOFs was used at a time. All spectrometers pointed to the focal spot of the beamline into which the various noble gas targets were introduced through a small needle in an effusive beam. The target gas was ionized by the FEL radiation and the generated photoelectrons were detected at the end of the flight tubes of the spectrometers by micro channel plate (MCP) detectors. The flight tubes of the spectrometers were designed with three retardation segments which allow for an energy resolution of $\frac{E}{\Delta E} \sim 1000$ in the whole photon energy range of this study from $38 \mathrm{eV}$ to $91 \mathrm{eV}$. The transmission was characterized for each spectrometer using single ionization emission. The total distance from the interaction region to the detector surface was $l=309 \mathrm{~mm}$ resulting in electron flight times of typically $100-300 \mathrm{~ns}$, depending on the kinetic energy of the electron and the applied retardation voltages. The MCP charge pulses were amplified and recorded by means of a multi-channel digitizer card system with a time resolution of up to $125 \mathrm{ps,} \mathrm{which} \mathrm{was} \mathrm{triggered}$ by the FLASH bunch clock with a bunch train repetition rate of $5 \mathrm{~Hz}$ and $10 \mathrm{~Hz}$ later on. Analogue signal traces of full FEL bunch trains were recorded, which in our case consisted typically of 30 and up to 50 micro pulses with a bunch repetition rate of $1 \mathrm{MHz}$, corresponding to a time-period of $1 \mu \mathrm{s}$ between bunches. In order to attribute the photon intensity of each FEL light pulse to the according electron time-of-flight spectra, the analogue signal of the gas monitor device (GMD) [34] delivered by the FLASH machine was recorded with the digitizer system as well. The average values of the ion-GMDs were used to correct the absolute values of the electron-GMD single-shot values. The experiments have been performed at the high-intensity beamlines BL2 and BL3 of the FLASH facility [31] for which the photon beam is focused to a spot size of about $30 \mu \mathrm{m}$ in diameter (FWHM). Taking single acquisitions of all experimental runs together, the average single pulse energy during 
a single acquisition session covers the range from $25 \mu \mathrm{J}$ up to $260 \mu \mathrm{J}$. Depending on the photon energy and with a typical pulse duration between $50 \mathrm{fs}$ and $100 \mathrm{fs}$, this corresponds to an irradiance in the range of $6 \cdot 10^{13}$ to $3 \cdot 10^{14} \mathrm{~W} / \mathrm{cm}^{2}$. Accordingly, the number of photons in a single FEL shot ranges from $4 \cdot 10^{12}$ to $5 \cdot 10^{13}$. The FEL wavelength was measured during the beam setup procedure by the machine operators prior to the experimental shift by means of a grating spectrometer in the FEL photon diagnostics section in the accelerator tunnel. Occasionally, it was re-checked during the shift for a selection of bunches in the pulse train. The accuracy of the average center of gravity of the photon energy was better than $0.5 \mathrm{eV}$ in all cases. Typically, the wavelength bandwidth of the spectral distribution was $1 \%-3 \%$ FWHM which, depending on the wavelength, corresponds to a photon energy bandwidth of $0.5 \mathrm{eV}$ to $2.5 \mathrm{eV}$, respectively.



Figure 1. Sketch of the experimental setup: The incoming FEL radiation ionizes the noble gas target which is introduced in the center of a spherical vacuum chamber in an effusive beam. A set of time-of-flight spectrometers are mounted in a plane perpendicular to photon beam which represents the dipole plane of the horizontally polarized FEL radiation. The anisotropy parameters of photoelectron emission determined in this work refer to this plane as indicated by the schematic angular distribution pattern (orange line). The signals of the MCP detector of each spectrometer are recorded with a multichannel digitizer system comprising up to 13 Acqiris DC 282 ADC modules. In our experiments, the analogue raw signal of the FLASH gas monitor device (GMD) was fed into one of the channels for intensity correlation. Using the $5 \mathrm{~Hz}$ and, later on, the $10 \mathrm{~Hz}$ pulse trigger of FLASH, traces of full bunch trains have been recorded using sampling rates of 2,4 , and 8 GS per second.

\section{Data Analysis}

In a single data acquisition, typically 4000 or 8000 traces of entire FEL bunch trains have been recorded. During the acquisition, the fast single-bunch resolved electron signal of the calibrated pulse energy monitor device GMD signal was recorded with the digitizer system and the mean pulse energy derived from the slow ion signal was noted for each acquisition. Hence, the pulse energy for each individual bunch could be derived and attributed to the corresponding rare gas photoelectron spectrum and a list of single-shot spectra sorted according to photon intensity could be generated. In the analysis afterwards, these traces have been cut into single pulse time-of-flight spectra and 
converted into kinetic energy spectra. For the photoline peak evaluation, different models for the background as well as several peak form functions (Gaussian, Lorentzian, and Voigt profile fitting as well as ROI-counting and combinations thereof) have been used with consistent results showing the stability of the evaluation [35].

Due to the narrow acceptance angle of our electron time-of-flight spectrometers, only relative intensities can be measured. These intensities depend on the quantum efficiency and the gain of each individual electron MCP detector. For intensity normalization, correction factors were introduced for each electron spectrometer and each acquisition. These factors were determined such that the intensities of signals of the primary ionization in the spectra match the angular distribution pattern of the one-photon single ionization described by equation (2) which is well known for all rare gases in the VUV photon energy range [1]. This procedure neglects higher order $\beta_{4}$-contributions in the angular distribution in the first ionization step $[18,28,29,36]$, which is a small effect and in addition affects only less than $30 \%$ of the primary ionization intensity (see below).

After applying the correction factors to the photoelectron intensities, the second step anisotropy parameters $\beta_{2}$ and $\beta_{4}$ were determined in a least-squares fitting procedure using equation (3) as a model function. To be able to discover possible effects on the results due to detector saturation at high pulse energy and poor statistics at low photon intensities, the evaluation was done for different intensity levels. For that, a set of sum spectra for different photon pulse energies was created by adding up single spectra belonging to certain intensity intervals by means of the GMD intensity list mentioned above. Correction factors derived from each of the sum spectra were applied to the photoline intensities of every sum spectra for $\beta$-parameter analysis. In this matrix-type evaluation (calibration for different photon intensities vs. $\beta$-parameter analysis for different photon intensities), the more recent experimental data have been evaluated together in a re-analysis of data sets of earlier beamtimes $[37,38]$. The new results turned out to be consistent over a large pulse energy region [35].

Furthermore, comparing acquisitions recorded at different photon energies and/or different target gases, we derived correction factors for different kinetic energies to account for transmission loss of the electron spectrometers.

\section{Results and discussion}

As an example for a typical time-of-flight spectrum and the underlying ionization scheme, figure 2 shows a photoelectron spectrum for argon at a photon energy of $38 \mathrm{eV}$. It comprises a sum of single shot spectra of the 10,000 most intense FEL pulses according to the GMD which have been recorded with one of the ToF spectrometers mounted at an angle of $34.5^{\circ}$ with respect to the horizontal polarization axis of the FEL.

By means of the kinetic energy, the four main features in the spectrum can be attributed to intermediate and final states of the photoionization. The most prominent photoline at a measured kinetic energy of $22.2 \mathrm{eV}$ represents the primary ionization which contains one-photon single ionization events as well as the first ionization step of sequential double ionization. The three lines at lower kinetic energies can be attributed to electrons emitted in the second step of two-photon ionization creating the three different doubly charged final states $\operatorname{Ar}^{2+}\left(3 p^{-2}\right)^{3} P\left(\mathrm{E}_{k i n}=10.1 \mathrm{eV}\right)$, $\operatorname{Ar}^{2+}\left(3 p^{-2}\right)^{1} D\left(\mathrm{E}_{k i n}=8.4 \mathrm{eV}\right)$, and $\operatorname{Ar}^{2+}\left(3 p^{-2}\right)^{1} S\left(\mathrm{E}_{k i n}=6.0 \mathrm{eV}\right)$. The total intensity of these three lines is in the order of about $30 \%$ of the primary signal which is a typical value for the ratio of the first-step and second-step yield in our experimental results. Clearly, sequential double ionization is a strong process under the applied conditions in terms of photon energy and photon intensity. Recorded signatures of processes involving two photons in the one ionization step which are not reported here are about two orders of magnitude weaker. Continuous photoelectron signatures of direct double ionization could not be observed. In our experiments, the FEL wavelength bandwidth did not allow to resolve the spin-orbit multiplets of either the singly charged intermediate state ${ }^{2} P$ or doubly charged final state ${ }^{3} P$. 


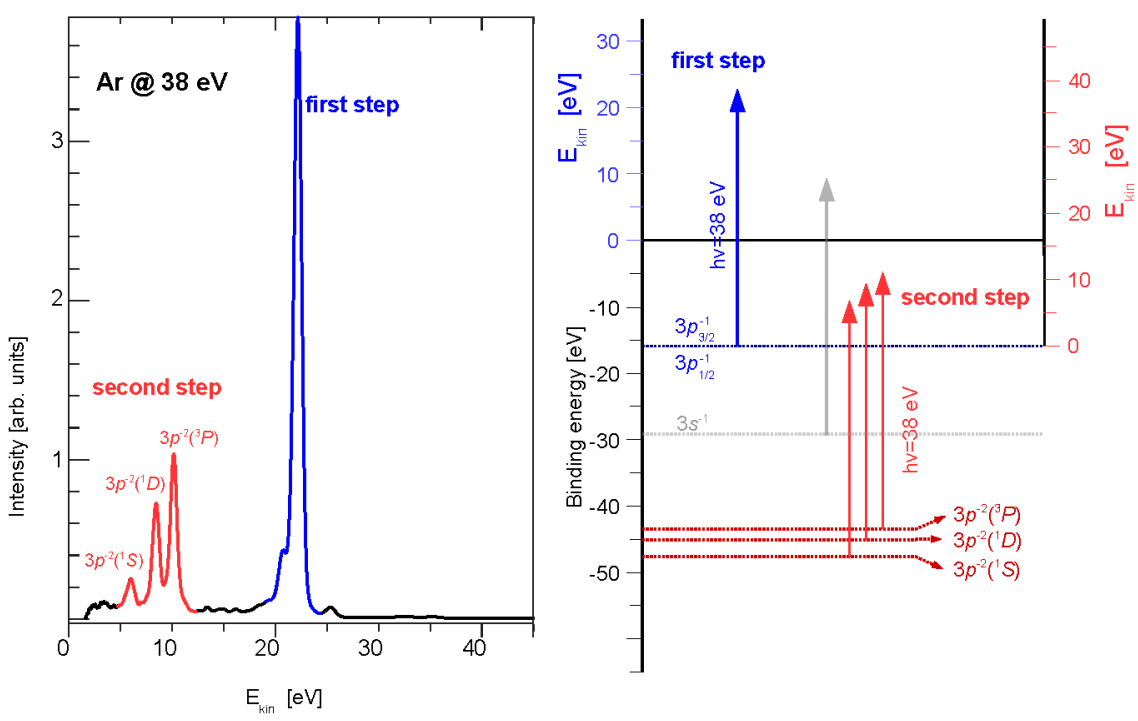

Figure 2. In the left panel, an example of a photoelectron spectrum over electron kinetic energy of argon recorded at a photon energy of $38 \mathrm{eV}$ is plotted. The most prominent photoelectron line (blue) represents the primary ionization creating the singly charged $\mathrm{Ar} 3 p^{-1}$ state, whereas the three photoelectron lines at lower kinetic energies are assigned to the doubly charged final states ${ }^{3} P,{ }^{1} D$, and ${ }^{1} S$ (red). The term scheme in the right panel illustrates the transitions of the two sequential ionization steps and the resulting electron kinetic energies.

\begin{tabular}{|c|c|c|c|c|}
\hline Target & State & Photon enery $[\mathrm{eV}]$ & $\beta_{2}$ & $\beta_{4}$ \\
\hline \hline $\mathrm{Ne}$ & $2 p^{-2}\left({ }^{3} P\right)$ & 47.5 & $0.79 \pm 0.10$ & $-0.21 \pm 0.10$ \\
\hline & & 52.3 & $0.80 \pm 0.13$ & $-0.20 \pm 0.13$ \\
\hline & & 61.2 & $1.11 \pm 0.14$ & $0.01 \pm 0.14$ \\
\hline & & 89.9 & $1.30 \pm 0.10$ & $-0.10 \pm 0.10$ \\
\hline & & 91.0 & $1.17 \pm 0.13$ & $-0.09 \pm 0.13$ \\
\hline & $2 p^{-2}\left({ }^{1} D\right)$ & 47.5 & $0.61 \pm 0.17$ & $-0.17 \pm 0.18$ \\
\hline & & 52.3 & $0.50 \pm 0.11$ & $-0.20 \pm 0.10$ \\
\hline & & 61.2 & $1.04 \pm 0.11$ & $-0.08 \pm 0.11$ \\
\hline & & 89.9 & $1.21 \pm 0.11$ & $-0.05 \pm 0.14$ \\
\hline $\mathrm{Ar}$ & $3 p^{-2}\left({ }^{3} P\right)$ & 38.0 & $1.70 \pm 0.15$ & $-0.30 \pm 0.15$ \\
\hline & & 47.5 & $1.25 \pm 0.16$ & $-0.22 \pm 0.16$ \\
\hline & & 61.2 & $0.70 \pm 0.19$ & $-0.21 \pm 0.17$ \\
\hline & & 91.0 & $1.45 \pm 0.19$ & $-0.27 \pm 0.19$ \\
\hline & $3 p^{-2}\left({ }^{1} D\right)$ & 38.0 & $1.86 \pm 0.16$ & $0.04 \pm 0.16$ \\
\hline & $3 p^{-2}\left({ }^{1} S\right)$ & 38.0 & $2.07 \pm 0.16$ & $0.21 \pm 0.16$ \\
\hline & & 61.2 & $1.20 \pm 0.20$ & $-0.11 \pm 0.16$ \\
\hline $\mathrm{Kr}$ & $4 p^{-2}\left({ }^{3} P\right)$ & 38.0 & $1.95 \pm 0.15$ & $-0.01 \pm 0.15$ \\
\hline & & 38.0 & $1.77 \pm 0.16$ & $-0.22 \pm 0.23$ \\
\hline & & 47.6 & $1.43 \pm 0.16$ & $-0.43 \pm 0.16$ \\
\hline & & 47.6 & $1.45 \pm 0.16$ & $0.19 \pm 0.25$ \\
\hline & & 61.6 & $0.88 \pm 0.24$ & $-0.24 \pm 0.19$ \\
\hline & & 78.5 & $1.06 \pm 0.24$ & $0.06 \pm 0.18$ \\
\hline
\end{tabular}

Table 1. Compilation of our experimental results for the $\beta$-parameters for the different final states of neon, argon and krypton. The errors given here account for the uncertainty resulting from determination of the detector intensity values in single shot spectra as well as the from averaging over the angular distribution parameters for different photon intensities. 
Our results for the second step ionization electron angular distributions of neon, argon and krypton compiled in table 1 are discussed in the following and compared to two recent theoretical studies which differ mainly in assumptions concerning coherency of excitation of the intermediate ion fine structure levels ${ }^{2} P_{1 / 2,3 / 2}$. In the density matrix and statistical tensor formalism used by Fritzsche et al. $[28,30,36]$ the excitation is considered completely incoherent (LSJ-coupling scheme), whereas Kheifets [29] used a model with completely coherent excitation of these levels (LS-coupling scheme). The latter work employs the graphical angular momentum coupling technique to derive analytical formulas for the $\beta$ parameters which contain the radial dipole matrix elements. These matrix elements are evaluated numerically using the random phase approximation with exchange.
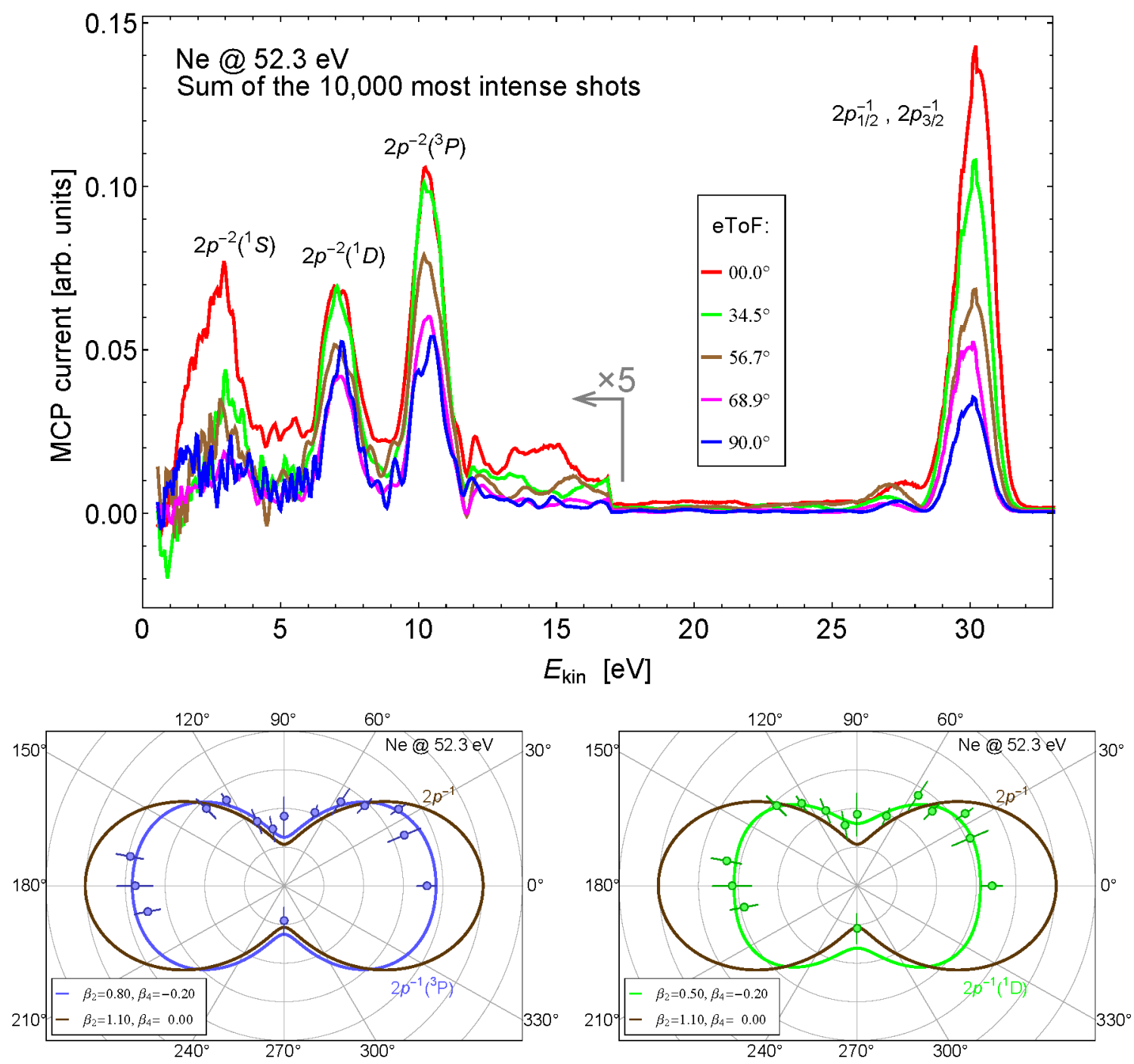

Figure 3. The top plot shows the spectra for selected spectrometers summed for the 10,000 most intense FEL shots. The high energy peak contains both the primary singly ionized Ne photoelectrons and the first step of the sequential double photoionization. The second step electrons are at 8 and $10 \mathrm{eV}$, respectively the ${ }^{1} \mathrm{D}$ and ${ }^{3} P$ electrons. The lower two polar plots show the angular distribution of the ${ }^{3} P$ (left) and ${ }^{1} D$ (right) photoelectrons in comparison to the distribution of the first step $2 \mathrm{p}^{-1}$ electron which was used for calibration. 


\section{Neon}

Acquisitions with neon as a target gas have been recorded at photon energies of 47.5, 52.3, 62,89 , and $91 \mathrm{eV}$. The upper panel of figure 3 shows sum spectra with corrected intensities of the most intense FEL shots for a selection of spectrometers used in the measurement at $h \nu=52.3 \mathrm{eV}$. Here, the spectra are magnified by a factor of five in the low kinetic energy region containing the photolines of the doubly charged states $\mathrm{Ne} 2 p^{-2}{ }^{3} \mathrm{P}$ and ${ }^{1} \mathrm{D}$. The ratio of intensities at diverse angles is different for the second step ionization signals compared to the primary ionization line which already indicates a different angular distribution for the intermediate and final states, respectively. The complete set of corrected intensities of the first and second step ionization photolines are presented in polar plots in the lower panels of figure 3, together with the results of the $\beta$ parameter analysis fit procedure and the resulting angular distribution patterns. The second step angular distribution patterns differ substantially from the first step pattern due to a considerably large negative value of the higher order $\beta$-parameter $\beta_{4}$ of around -0.2 . In figure 4 , the derived $\beta$ values of all neon measurements (see tab.1) are displayed together with the theoretical calculations for comparison. The theoretical data show a smooth change in $\beta_{2}$ with increasing photon energy - like in the single ionization - and almost constant values for $\beta_{4}$, but different values for the three doubly charged final states. The agreement with our experimental results is comparably good for the $\beta_{2}$ values of both theoretical methods. With respect to the $\beta_{4}$ parameter, the predicted positive values for ${ }^{1} D$ close to zero are in contrast to our measurements. The experimental values for the ${ }^{3} P$ final state agree better with the results of Kheifets [29] for low photon energies, whereas for higher photon energies there is better accordance with the calculation of Fritzsche et al. [28]. In addition, the small difference of $\beta_{4}$ parameters for ${ }^{1} D$ and ${ }^{3} P$ in the latter theoretical model corresponds better to the measured values. Energetically the ${ }^{1} S$ final state is superimposed by the $2 \mathrm{~s}$ single-photionization line. A meaningful angular distribution analysis was therefore not possible.
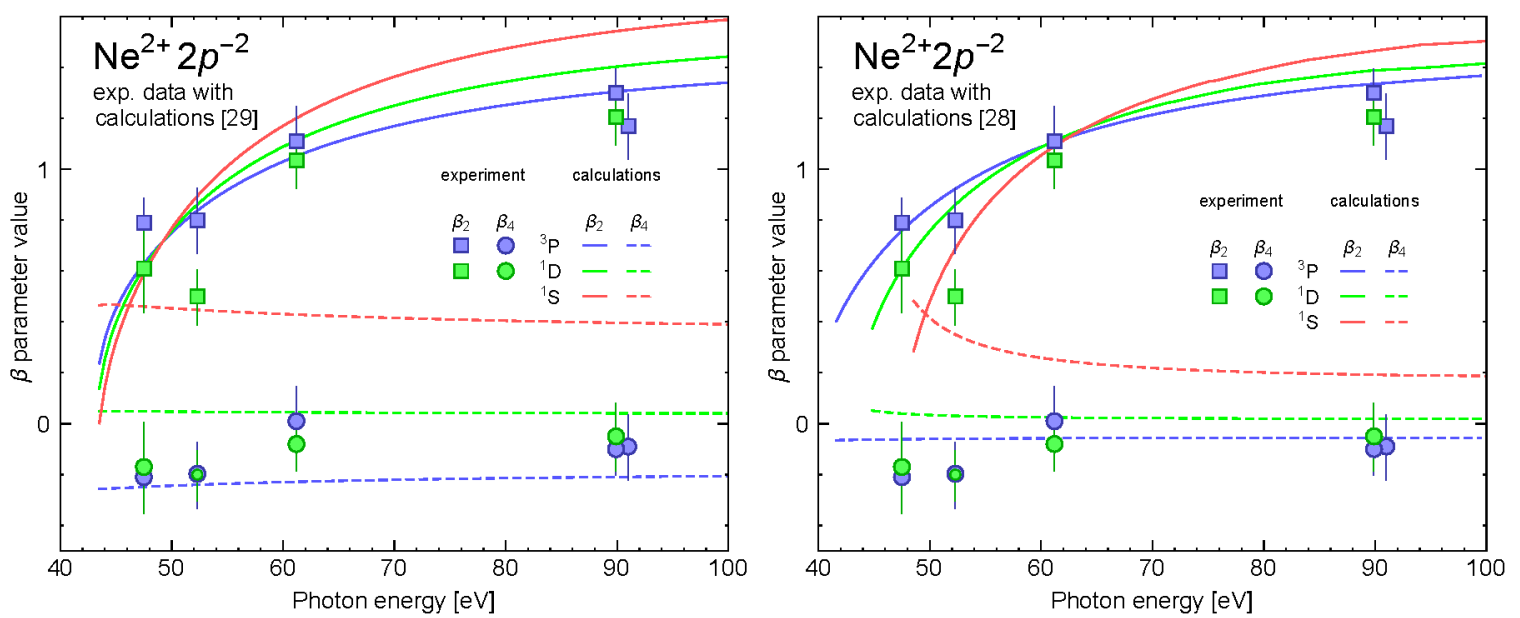

Figure 4. Anisotropy parameters $\left(\beta_{2}\right)$ (squares) and $\left(\beta_{4}\right)$ (circles) for the second step of sequential two-photon double ionization of neon as a function of photon energy. Our results are compared to the calculations for $\left(\beta_{2}\right)$ (solid lines) and $\left(\beta_{4}\right)$ (dashed lines) of Kheifets [29] (left panel) and Fritzsche et al. [28] (right panel).

\section{Argon}

For argon, angular distribution measurements have been performed at photon energies of $38,47.5,62$, and $91 \mathrm{eV}$. In figure 5, the experimentally determined $\beta$-parameter values are shown together with recent calculations [28, 29]. Compared to neon, these calculated curves show a larger variation of the $\beta$-parameters along the photon energy. The curves of $\beta_{2}$ for the three final 
states, $\mathrm{Ar}^{2+}\left(3 p^{-2}\right)^{3} P, \mathrm{Ar}^{2+}\left(3 p^{-2}\right)^{1} D$ and $\mathrm{Ar}^{2+}\left(3 p^{-2}\right)^{1} S$ run quite uniformly through a minimum, whereas the $\beta_{4}$ values vary very differently due to the anisotropic interactions, especially in the region of the minimum in the $\beta_{2}$ theory curves. It is known from single ionization, that the minimum in the $\beta$-parameter is slightly shifted towards higher photon energies compared to the related Cooper minimum position of the cross-section at around $h \nu=50 \mathrm{eV}$ [1]. For the double ionization, with both theoretical methods shown in figure 5 the $\beta_{2}$ minima positions are calculated to be at significantly lower photon energies than the single ionization $\beta_{2}$ minimum. However, we assume that the difference between the radial wave function overlap conditions of the first and the second ionization step by ejection of only one shell electron is small. Therefore, all the calculated double ionization curves are shifted to coincide with the single ionization $\beta_{2}$-Cooper minimum. This shift of 5 and $10 \mathrm{eV}$, respectively, (see figure 5) introduces no new physics, moreover, it is just a correction of the theoretical data which is still in the range of the expected accuracy of the theoretical models in terms of the energy scale which the authors amount to be within a few electron volts ( $4 \mathrm{eV}$ in the case of the calculation of $\mathrm{A}$. Kheifets).

The agreement between our experimental data and the shifted theory curves is rather moderate except for the lowest photon energy of $38 \mathrm{eV}$, for which the experimental values of $\beta_{2}$ and $\beta_{4}$ coincide very well with the different values of the three final states $\operatorname{Ar}^{2+}\left(3 p^{-2}\right)^{3} P, \mathrm{Ar}^{2+}\left(3 p^{-2}\right)^{1} D$ and $\mathrm{Ar}^{2+}\left(3 p^{-2}\right)^{1} S$ from both calculations. The region of the Cooper minimum is particularly interesting, where especially the angular momentum coupling theory [29] predicts a tremendous difference for the $\beta_{4}$ values for the ${ }^{3} P,{ }^{1} D$, and ${ }^{1} S$ states. Whereas for this theoretical model there is good agreement with the calculated $\beta_{2}$-values, we cannot confirm a very pronounced anisotropy in the angular emission pattern with a large negative value of $\beta_{4}=-1.7$. Instead, we measured a rather isotropic distribution with only small lobes in horizontal and vertical direction, which is more consistent with the results of the calculation of Fritzsche et al. [28].

Generally, in the case of argon the $3 s$ single ionization line position practically coincides with the ${ }^{1} \mathrm{D}$ 2-photon-line which precluded reliable ${ }^{1} \mathrm{D}$ data analysis.


Figure 5. Anisotropy parameters $\beta_{2}$ (squares) and $\beta_{4}$ (circles) for the second step of sequential two-photon double ionization of argon as a function of photon energy. Our results are compared to the calculations for $\beta_{2}$ (solid lines) and $\beta_{4}$ (dashed lines) of Kheifets [29] (left panel) and Fritzsche et al. [28] (right panel). The theory curves are shifted by $10 \mathrm{eV}$ and 5 eV, respectively, towards larger photon energy (see text). The experimental data points for ${ }^{3} P$ and ${ }^{1} S$ at $h \nu=62 \mathrm{eV}$ lie on top of each other in this axis scale.

\section{Krypton}

Regarding the sequential ionization of krypton, we present data for the photon energies 38 , $47.5,62,78.5 \mathrm{eV}$ for the ${ }^{3} P$ doubly charged final state only (see figure 6 ). Similar to the argon case, 
the krypton single ionization cross section shows a Cooper minimum with an according reflection in the single ionization $\beta$-curve at a photon energy of $84 \mathrm{eV}$, in this case $[1]$. Again, the $\beta$-minima in the calculations for the second step ionization deviate from this position and theory curves were shifted accordingly, as depicted in figure 6 . In the photon energy range on the plots, both theoretical models predict a decrease of $\beta_{2}$ with increasing photon energy starting from a value around 2, which is reproduced by our experimental data up to $62 \mathrm{eV}$. For $\beta_{4}$ the calculations do not show variations around the minimum as large as for $\mathrm{Ar}$, in fact the $\beta_{4}$ values of the theoretical description used in [30] are basically close to zero over the whole plot range. A rather small higher order anisotropy effect is in accordance with our data. Remarkably, the experimental value for $\beta_{2}$ at $78.5 \mathrm{eV}$ in the region of the Cooper minimum deviates strongly from both calculations. The correspondingly measured angular distribution exhibits pronounced emission probability in the horizontal direction, whereas according to the calculations the emission pattern should be either almost completely isotropic, as in the model equivalent to the LSJ-coupling scheme [30], or show predominant intensity at angles around $45^{\circ}$ with respect to horizontal or vertical directions as derived with the graphical angular momentum coupling technique.
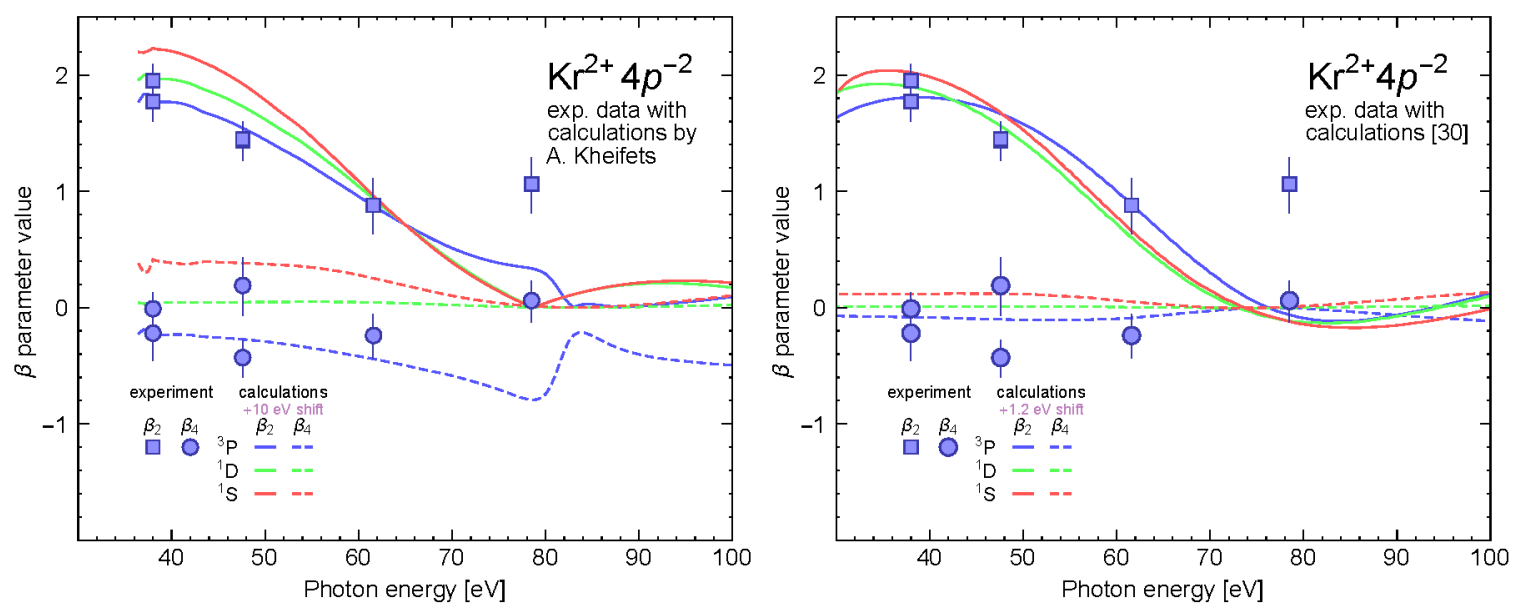

Figure 6. Anisotropy parameters $\beta_{2}$ (squares) and $\beta_{4}$ (circles) for the second step of sequential two-photon double ionization of krypton as a function of photon energy. Our results are compared to the calculations for $\beta_{2}$ (solid lines) and $\beta_{4}$ (dashed lines) of Kheifets (left panel) and Fritzsche et al. [30] (right panel). The theory curves are shifted by $10 \mathrm{eV}$ and $1.2 \mathrm{eV}$, respectively, towards larger photon energy (see text).

\section{Conclusion (and Outlook)}

Our report shows that FEL photon sources are able to induce strong sequential two-photon double ionization processes in rare gases which are almost as intense as the single ionization in the photon energy range between $38 \mathrm{eV}$ and $91 \mathrm{eV}$. For a number of different photon energies, we measured the angular distribution patterns of photoelectrons emitted in the second ionization step. The anisotropy parameters $\beta_{2}$ and $\beta_{4}$ of these angular distributions could be derived for the doubly charged final states ${ }^{1} S,{ }^{1} \mathrm{D}$, and mainly ${ }^{3} \mathrm{P}$. The comparison with two different theory calculations showed altogether reasonable agreement, but the results do not allow favoring one or the other in general. However, based on our results there is qualitatively no experimental indication for a change of the parameter $\beta_{4}$ of the $\mathrm{Ar}{ }^{3} \mathrm{P}$ state in the region of the Cooper minimum as large as predicted by the LS coupling approximation of [29]. To confirm our findings and to improve quantitative significance, further measurements at a number of photon energies in the Cooper minimum region are necessary. The role of Cooper minima for sequential ionization processes and its impact on the angular distributions of all involved steps is especially important with respect to a breakdown of the dipole approximation in the non-linear regime [39]. A future investigation of 
this kind can benefit from the recent prosperity in FEL evolution, especially in terms of wavelength tunabilty. For example, the seeded FEL source at FERMI [40] as well as FLASH2 [41, 42] with variable gap undulators coming to operation soon allow fast wavelength changes which will facilitate scanning over a wavelength interval. Furthermore, new developments in photon diagnostics, like a FEL radiation polarization monitor [43] very similar to our experimental setup, can support these efforts in terms of experimental accuracy as well fast handling and processing of large amounts of data in order to map out the details of electron angular distribution effects in multi-photon ionization.

\section{Acknowledgements}

We dedicate this work to the late Professor Doktor Uwe Becker who initiated these investigations. Furthermore, the authors are indebted to N.M. Kabachnik, A.N. Grum-Grzhimailo, S. Fritzsche, and E.V. Gryzlova for tremendous discussions regarding the theory and mathematical models of the investigated processes. MB thanks B. Langer, D. Rolles, R. Püttner, B. Lohmann, M. Martins, A. Rudenko, and R. Moshammer for support and fruitful discussions. We gratefully acknowledge the excellent user support at FLASH by R. Treusch, S. Düsterer, G. Brenner, M. Kuhlmann, K. Tiedtke, S.Toleikis, H. Wabnitz.

\section{Funding}

This project was funded by the BMBF grants under promotion code numbers 05K13RK1, 05KS4EB1/3 and 05KS7EBA as well as the CFEL Advanced Study Group. The work was supported by the Hesse State Initiative for the Development of Scientific and Economic Excellence (LOEWE) in the LOEWE-Focus Electron Dynamics of Chiral Systems (ELCH). MI acknowledges financial support by the Volkswagen foundation within the Peter Paul Ewald fellowship.

\section{References}

[1] Becker, U.; Shirley, D. A. VUV and Soft X-Ray Photoionization; Plenum Press, New York, 1996.

[2] Becker, U.; Shirley, D. Physica Scripta 1990, 1990, 56.

[3] Becker, U. Journal of electron spectroscopy and related phenomena 1995, 75, 23-34.

[4] Avaldi, L.; Huetz, A. Journal of Physics B: Atomic, Molecular and Optical Physics 2005, 38, S861.

[5] Åberg, T. Atomic Inner Shell Processes, Vol. I. Ionization and Transition Probabilities. 1975.

[6] Åberg, T.; Howat, G. Handbuch der Physik vol 31 ed S Flügge and W Mehlhorn. 1982.

[7] Viefhaus, J.; Grum-Grzhimailo, A.; Kabachnik, N.; Becker, U. Journal of Electron Spectroscopy and Related Phenomena 2004, 141, 121 - 126.

[8] Viefhaus, J.; Cvejanović, S.; Langer, B.; Lischke, T.; Prümper, G.; Rolles, D.; Golovin, A. V.; GrumGrzhimailo, A. N.; Kabachnik, N. M.; Becker, U. Phys. Rev. Lett. 2004, 92, 083001.

[9] Viefhaus, J.; Braune, M.; Korica, S.; Reinköster, A.; Rolles, D.; Becker, U. Journal of Physics B: Atomic, Molecular and Optical Physics 2005, 38, 3885.

[10] Amusia, M. Y.; Kilin, V.; Ehresmann, A.; Schmoranzer, H.; Schartner, K.-H. Journal of Physics B: Atomic, Molecular and Optical Physics 1993, 26, 1281.

[11] Ackermann, W.; Asova, G.; Ayvazyan, V.; Azima, A.; Baboi, N.; Bähr, J.; Balandin, V.; Beutner, B.; Brandt, A.; Bolzmann, A. Nature Photonics 2007, 1, 336-342.

[12] Allaria, E.; Appio, R.; Badano, L.; Barletta, W.; Bassanese, S.; Biedron, S.; Borga, A.; Busetto, E.; Castronovo, D.; Cinquegrana, P.; et al., Nature Photonics 2012, 6, 699-704.

[13] Ishikawa, T.; Aoyagi, H.; Asaka, T.; Asano, Y.; Azumi, N.; Bizen, T.; Ego, H.; Fukami, K.; Fukui, T.; Furukawa, Y.; et al., Nature Photonics 2012, 6, 540-544.

[14] Emma, P.; Akre, R.; Arthur, J.; Bionta, R.; Bostedt, C.; Bozek, J.; Brachmann, A.; Bucksbaum, P.; Coffee, R.; Decker, F.-J.; et al., Nature Photonics 2010, 4, 641-647. 
[15] Rudek, B.; Son, S.-K.; Foucar, L.; Epp, S. W.; Erk, B.; Hartmann, R.; Adolph, M.; Andritschke, R.; Aquila, A.; Berrah, N.; et al., Nature Photonics 2012, 6, 858-865.

[16] Richter, M.; Bobashev, S.; Sorokin, A.; Tiedtke, K. Journal of Physics B: Atomic, Molecular and Optical Physics 2010, 43, 194005.

[17] Berrah, N.; Bozek, J.; Costello, J.; Düsterer, S.; Fang, L.; Feldhaus, J.; Fukuzawa, H.; Hoener, M.; Jiang, Y.; Johnsson, P.; et al., Journal of Modern Optics 2010, 57, 1015-1040.

[18] Rudenko, A.; Jiang, Y.; Kurka, M.; Kühnel, K.; Foucar, L.; Herrwerth, O.; Lezius, M.; Kling, M.; Schröter, C.; Moshammer, R.; et al., Journal of Physics B: Atomic, Molecular and Optical Physics 2010, 43, 194004.

[19] Kurka, M.; Rudenko, A.; Foucar, L.; Kühnel, K.; Jiang, Y.; Ergler, T.; Havermeier, T.; Smolarski, M.; Schössler, S.; Cole, K.; et al., Journal of Physics B: Atomic, Molecular and Optical Physics 2009, 42, 141002 .

[20] Hikosaka, Y.; Fushitani, M.; Matsuda, A.; Tseng, C.-M.; Hishikawa, A.; Shigemasa, E.; Nagasono, M.; Tono, K.; Togashi, T.; Ohashi, H.; et al., Physical review letters 2010, 105, 133001.

[21] Haber, L. H.; Doughty, B.; Leone, S. R. Physical Review A 2009, 79, 031401.

[22] O'Keeffe, P.; Bolognesi, P.; Mihelic, A.; Moise, A.; Richter, R.; Cautero, G.; Stebel, L.; Sergo, R.; Pravica, L.; Ovcharenko, E.; et al., Physical Review A 2010, 82, 052522.

[23] O'Keeffe, P.; Mihelic, A.; Bolognesi, P.; Zitnik, M.; Moise, A.; Richter, R.; Avaldi, L. New Journal of Physics 2013, 15, 013023.

[24] Cooper, J. W. Phys. Rev. 1962, 128, 681-693.

[25] Sukhorukov, V.; Lagutin, B.; Petrov, I.; Schmoranzer, H.; Ehresmann, A.; Schartner, K.-H. Journal of Physics B: Atomic, Molecular and Optical Physics 1994, 27, 241.

[26] Ehresmann, A.; Vollweiler, F.; Schmoranzer, H.; Sukhorukov, V.; Lagutin, B.; Petrov, I.; Mentzel, G.; Schartner, K.-H. Journal of Physics B: Atomic, Molecular and Optical Physics 1994, $27,1489$.

[27] Adam et al., M. Journal of Electron Spectroscopy and Related Phenomena 1985, 36, 377 - 390.

[28] Fritzsche, S.; Grum-Grzhimailo, A. N.; Gryzlova, E. V.; Kabachnik, N. M. Journal of Physics B: Atomic, Molecular and Optical Physics 2008, 41, 165601.

[29] Kheifets, A. S. Journal of Physics B: Atomic, Molecular and Optical Physics 2007, 40, F313.

[30] Fritzsche, S.; Grum-Grzhimailo, A. N.; Gryzlova, E. V.; Kabachnik, N. M. Journal of Physics B: Atomic, Molecular and Optical Physics 2009, 42, 145602.

[31] Tiedtke, K.; Azima, A.; Von Bargen, N.; Bittner, L.; Bonfigt, S.; Düsterer, S.; Faatz, B.; Frühling, U.; Gensch, M.; Gerth, C.; et al., New journal of physics 2009, 11, 023029.

[32] Yang, C. N. Phys. Rev. 1948, 74, 764-772.

[33] Balashov, V. V.; Grum-Grzhimailo, A. N.; Kabachnik, N. M. Polarization and Correlation Phenomena in Atomic Collisions: A Practical Theory Course; New York: Plenum, 2000.

[34] Tiedtke, K.; Feldhaus, J.; Hahn, U.; Jastrow, U.; Nunez, T.; Tschentscher, T.; Bobashev, S.; Sorokin, A.; Hastings, J.; Möller, S.; et al., Journal of Applied Physics 2008, 103, 094511.

[35] Hartmann, G. Coherence effects of diatomic homonuclear molecules and sequential two-photon processes of noble gases in the photoionization. Ph.D. thesis, 2014.

[36] Fritzsche, S.; Grum-Grzhimailo, A. N.; Gryzlova, E. V.; Kabachnik, N. M. Journal of Physics B: Atomic, Molecular and Optical Physics 2008, 41, 199801.

[37] Braune, M.; Reinköster, A.; Viefhaus, J.; Lohmann, B.; Becker, U. ICPEAC: 25th Int. 2007.

[38] Braune, M.; Lischke, T.; Meissner, A.; Ilchen, M.; Deinert, S.; Viefhaus, J.; Knie, A.; Becker, U. ICPEAC: XXVII. 2007.

[39] Grum-Grzhimailo, A.; Gryzlova, E.; Meyer, M. Journal of Physics B: Atomic, Molecular and Optical Physics 2012, 45, 215602.

[40] Allaria, E.; Castronovo, D.; Cinquegrana, P.; Craievich, P.; Dal Forno, M.; Danailov, M.; D'Auria, G.; Demidovich, A.; De Ninno, G.; Di Mitri, S.; et al., Nature Photonics 2013, 7, 913-918.

[41] Faatz, B.; Baboi, N.; Ayvazyan, V.; Balandin, V.; Decking, W.; Duesterer, S.; Eckoldt, H.-J.; Feldhaus, J.; Golubeva, N.; Honkavaara, K.; et al., Nuclear Instruments and Methods in Physics Research Section A: Accelerators, Spectrometers, Detectors and Associated Equipment 2011, 635, S2-S5.

[42] Honkavaara, K.; Ackermann, S.; Ayvazyan, V.; Baboi, N.; Balandin, V.; Decking, W.; Düsterer, S.; Eckoldt, H.; Faatz, B.; Felber, M.; et al., Status of the FLASH II Project. 2013.

[43] Allaria, E.; Diviacco, B.; Callegari, C.; Finetti, P.; Mahieu, B.; Viefhaus, J.; Zangrando, M.; De Ninno, G.; Lambert, G.; Ferrari, E.; et al., Physical Review X 2014, 4, 041040. 